\title{
The Acoustic Reflex for Filtered Broadband Stimuli: A Lesser Contribution of the Lower Frequency Neurons
}

\author{
Tetsuaki Kawase, Hiroshi Hidaka, Masaki Ogura, Sho \\ Hashimoto and TOMONORI TAKasaka \\ Department of Otolaryngology, Tohoku University School of \\ Medicine, Sendai 980-8574
}

\begin{abstract}
Kawase, 'T., Hidaka, H., Ogura, M., Hashimoto, S. and Takasaka, T. The Acoustic Reflex for Filtered Broadband Stimuli: A Lesser Contribution of the Lower Frequency Neurons. Tohoku J. Exp. Med., 1998, 185 (2), 131-137 — The effects of the cut-off frequency of the filtered broadband stimuli on the human acoustic reflex $(\mathrm{AR})$ were examined to observe the relation between the area of excitation in the cochlea and the AR response. The results obtained have indicated that all the input from the cochlear region does not equally contribute to trigger the AR equally; i.e., there is a lesser contribution from the frequency region below $700 \mathrm{~Hz}$. acoustic reflex; filtered noise; input mechanism; human (C) 1998 Tohoku University Medcial Press
\end{abstract}

As a part of the efferent control system in the auditory pathway, middle ear muscles can affect sound transmission to the inner ear. Although it is well known that the contraction of middle ear muscles (MEMs) in response to loud sound occurs in many species (Klockhoff 1961; Carmel and Starr 1963; Borg 1972; Borg et al. 1984), the mechanism of this acoustic reflex (AR) is not fully known. It has been hypothesized that the spread of excitation through the cochlear neurons is possibly an important factor in this mechanism (Kobler et al. 1992; Kawase et al. 1997). In other words, the acoustic reflex is triggered by the summed activity of afferent neurons derived from different cochlear frequency regions. It can be hypothesized that the acoustic reflex is provoked when the summed activity of afferent neurons reaches a point above some particular level (threshold). However, there has been little discussion as to whether the input from all portions of the cochlear region contribute to the reflex equally, in other words, if the contribution of the acoustic nerve differs according to its characteristic frequency (CF).

It is well known that broadband noise (BBN) can lower the acoustic reflex thresholds (ART) (Deutsch 1972; Peterson and Liden 1972; Margolis and Popelka

Received April 7, 1998; revision accepted for publication June 30, 1998.

Address for reprints: Tetsuaki Kawase, M.D., Department of Otolaryngology, Tohoku

University School of Medicine, 1-1 Seiryomachi, Aoba-ku, Sendai 980-8574, Japan.

e-mail: kaw@cctu.cc.tohoku.ac.jp 
1975). In order to excite not only the neurons innervated around the CF region for the stimulus but also those located in the off-CF region, a high level stimulus would be necessary to excite the high threshold parts (i.e., tail part of the tuning curve) of the off-CF neurons when using the narrow band stimuli to elicit the AR. On the contrary, since BBN stimulates the auditory-nerve fibers located in a broad area along the cochlear partition at the same time, it is possible that a sufficient number of auditory neurons is excited to the extent necessary to elicit the AR even when a lower level of BBN is presented. A lower ART for BBN also seems to be in accordance with the above-mentioned theory for AR input. In the present study, the effects of the cut-off frequency of the filtered broadband stimuli on the $\mathrm{AR}$ were examined to elucidate the relation between the excitation area and the $\mathrm{AR}$ response. The results are discussed in connection with the acoustic input of the reflex arc.

\section{Materials and Methods}

Six ears from 4 healthy cases ( 3 males and 1 female, with a mean age of 26.3 years) were examined. Inspection of the tympanic membrane and tympanometric measurements revealed no pathologic findings in any of the subjects. Standard tonal audiometry performed in a soundproof room indicated no elevation of pure-tone thresholds at the standard test frequencies of $0.25,0.5$, $1,2,4$ and $8 \mathrm{kHz}$ (within $20 \mathrm{~dB}$ hearing level at all tested frequencies) in any subjects. Sound stimuli to evoke the AR were presented in the ear opposite to that used to record the AR (contralateral reflex).

The AR of the MEMs was measured by means of an impedance audiometer (Teledyne Avionics, model TA-2C, Charlottesville, VA, USA) with a probe tone of $226 \mathrm{~Hz}$. In this device, impedance changes are represented by a compliance value which is shown by an equivalent volume of air $(\mathrm{ml})$ and can be monitored by a recorder as well as by needle deflection.

As stimuli to elicit the AR, white and low-frequency weighted noises (weighted noise) were used in the present study, and the effects of the cut-off frequency of a high-pass filter on the ART were observed. The Fast Fourier Transform (FFT) analysis of these stimuli (electrical output from the generator) is shown in Fig. 1. These broadband stimuli were filtered by a high- and low-pass digital filter (PF1, Tucker Davis, Gainesville, FL, USA) (49.6 dB/oct), and presented through a earphone (RION AD-20, Tokyo). Calibration on a standard $6 \mathrm{ml}$ coupler indicated that the frequency response of this earphone was very flat from 100 to $2000 \mathrm{~Hz}$ and that the sensitivity was reduced by about $2-6 \mathrm{~dB}$ from 2000 to $8000 \mathrm{~Hz}$. Cut-off frequencies for the high-pass stimuli used in the present study were $4000,2830,2000,1410,1000,710,500,355,250,175$ and $125 \mathrm{~Hz}$. In addition, the effects of the cut-off frequency of the high-pass filter on the ART were observed in the weighted noise filtered by a low-pass filter at a frequency of $2000 \mathrm{~Hz}(2-\mathrm{kHz}$ low-pass weighted noise), as well. In this series, the cut-off 


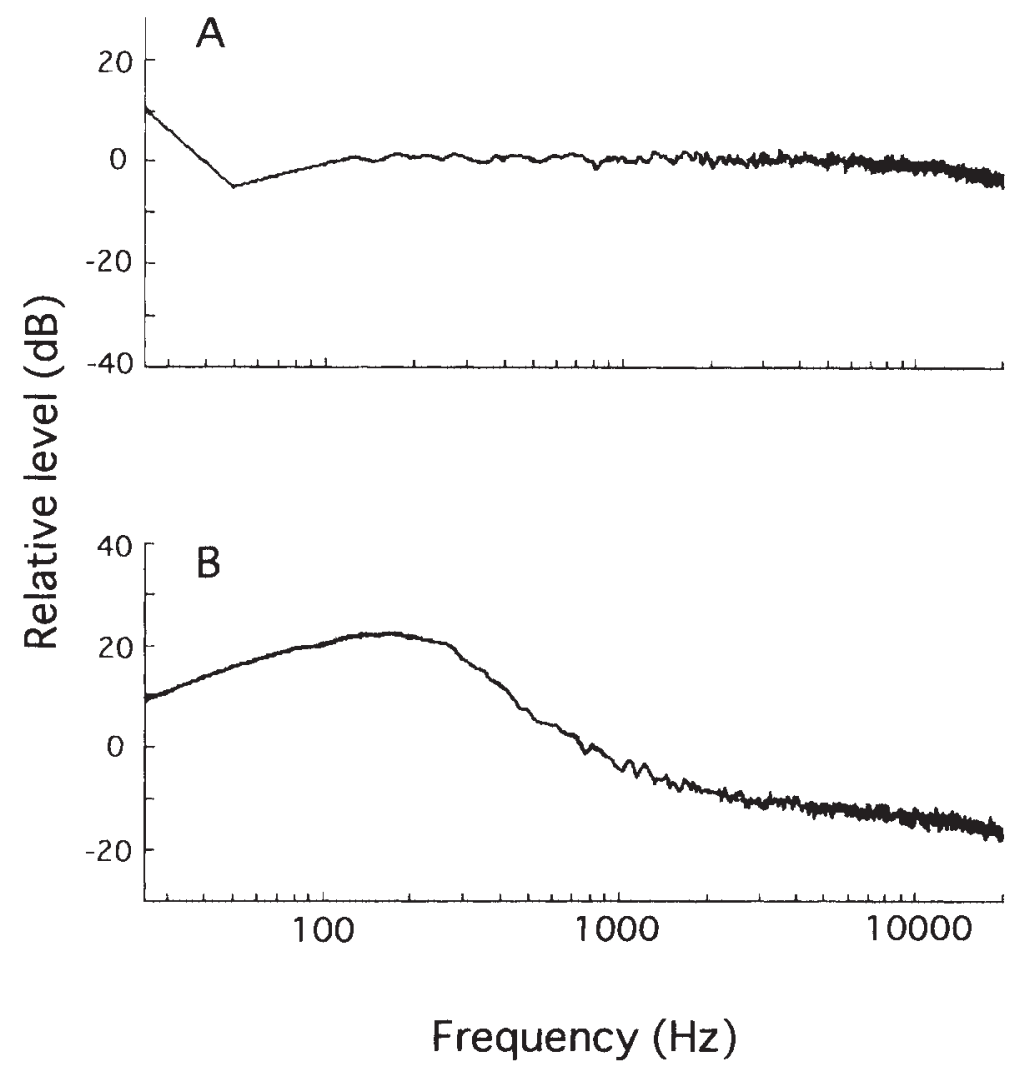

Fig. 1. FFT analysis of white noise (A) and low frequency weighted noise (B) (electrical output from the generator).

frequencies on the lower frequency side were varied from $1410 \mathrm{~Hz}$ to $125 \mathrm{~Hz}$.

Stimuli (duration: 1 second; rise-fall time: 50 mseconds) were presented at a repetition rate of $1 / 10$ seconds. First, the sound level of the contralateral stimuli was changed by 5 -dB steps from a level below its ART. The sound level was then elevated by 1-dB steps near the level of the ART, and the ART was defined as the sound level which causes minimal detectable impedance change as shown by the needle deflector. The sound pressure levels produced by the receiver were calibrated using a standard coupler by means of a B \& $\mathrm{K}$ measuring amplifier (Brüel \& Kjær, Nærum, Denmark) with a 1/4-inch microphone. As the actual sound pressure level in the ear canal of each subject was not measured, the sound level presented to each ear was expressed as the values calibrated in the coupler.

All parts of the present study were performed in accordance with the guidelines of the Declaration of Helsinki.

\section{RESULTS}

In Fig. 2, typical results of the effects of the cut-off frequency on the ART for three different conditions in one ear are presented. In Figs. $2 \mathrm{~A}$ and $\mathrm{B}, \mathrm{ART}$ s are expressed by the attenuation level and absolute sound pressure level, respectively. As long as the cut-off frequency was not lower than $700 \mathrm{~Hz}$, the ART expressed both by the attenuation level of the noise and by the sound pressure level 


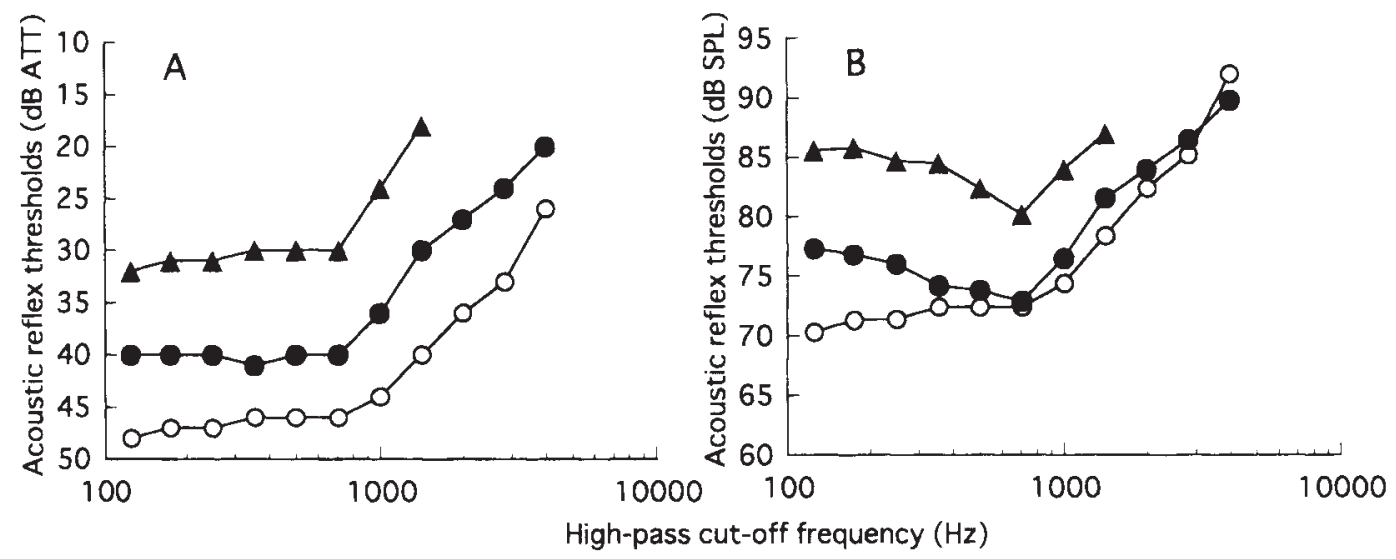

Fig. 2. Typical results of the effects of the cut-off frequency on the ART for three different types of noise (white, weighted and low-pass weighted noises). ARTs are expressed by the attenuation level and absolute sound pressure level in $\mathrm{A}$ and $\mathrm{B}$, respectively. ( $\mathrm{O}$, white; $\boldsymbol{\bullet}$, weighted; $\boldsymbol{\Delta}, 2 \mathrm{kHz}$ low-pass weighted)

decreased as the cut-off frequency decreased. However, no effects of broadening of the passed frequency on the ART were observed below $700 \mathrm{~Hz}$ (Fig. 2A). When expressed by the absolute level (Fig. 2B), ARTs elevated with the decrease in the cut-off frequency by reflecting the frequency character of the weighted noise. Basically, no differences were observed between the three types of noises, i.e., white, weighted, and $2 \mathrm{kHz}$ low-pass weighted noises; that is, the results indicate that an increase of the low-frequency sound power does not affect the ART significantly.

The same trend was observed in the other five ears, as well. In Fig. 3, the results for all the subjects are plotted. The reduction of the cut-off frequency below $500-700 \mathrm{~Hz}$ does not seem to affect the ART significantly.

\section{Discussion}

The effects of the cut-off frequency, mostly in relation to the critical band, have been investigated by many authors (Flottorp et al. 1971; Djupesland and Zwislocki 1973; Popelka et al. 1976). However, such effects have not been discussed in relation to the possible contribution of the cochlear nerve according to the cochlear frequency region. The present study was basically motivated by a previous report by Margolis et al. (1980). In that study, using high- and low-pass filtered noise, it was found that the energy below approximately $700 \mathrm{~Hz}$ did not appreciably contribute to the sensitivity of the reflex for BBN activators. Although their results may indicate the possible lesser contribution of nerve fibers originating in the cochlear frequency region below $700 \mathrm{~Hz}$, the following possibility also appeares to be plausible. As is well known, hearing sensitivity in man has decreased below $500 \mathrm{~Hz}$ (Dadson and King 1952; Robinson and Dadson 1956). Therefore, when using filtered white noise, sound energy in the lower frequency region is possibly significantly smaller than that in the upper frequency region in 


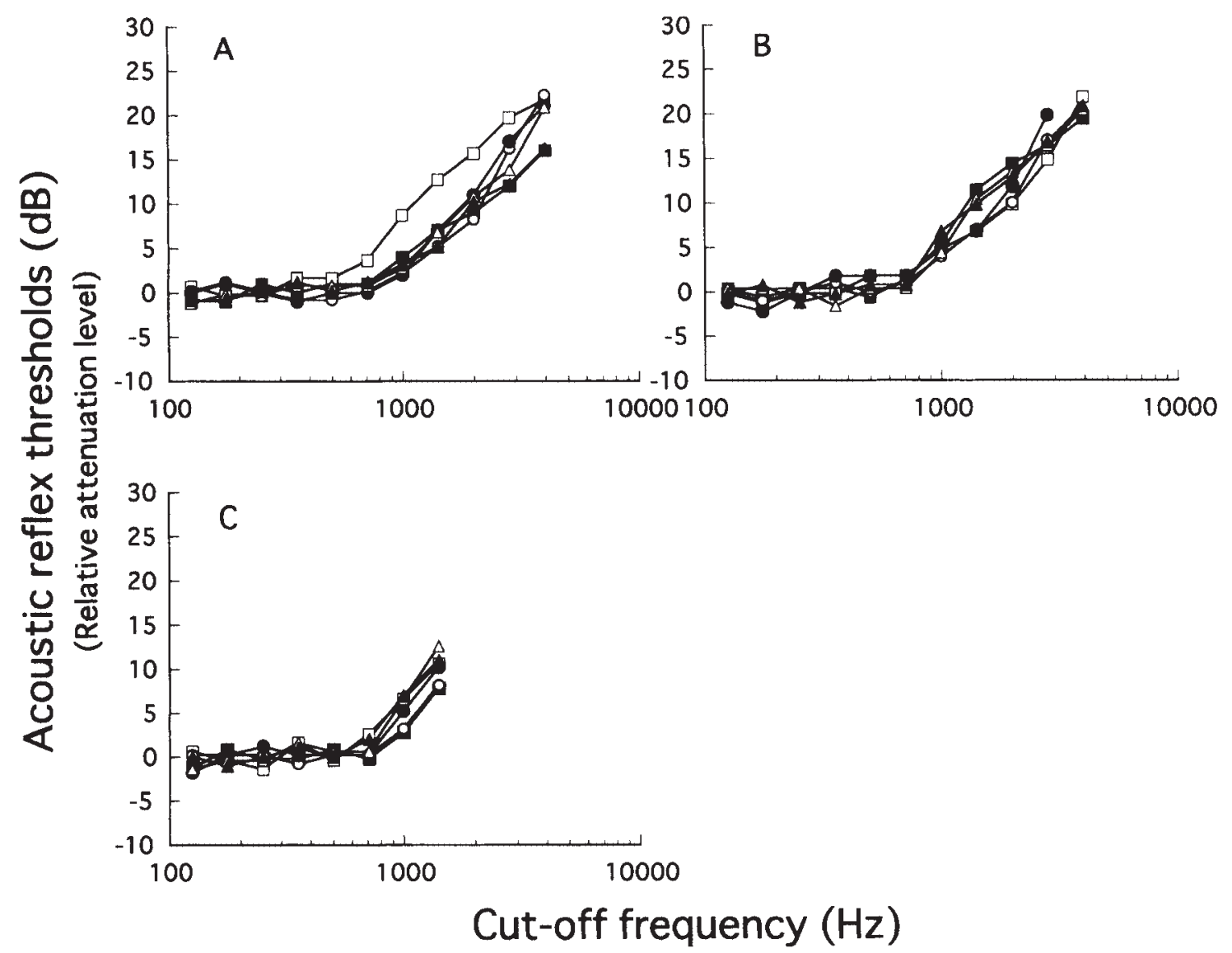

Fig. 3. The results for all subjects. ART levels are expressed by the relative attenuation level normalized by the average attenuation level of ARTs for the filtered noises cut off at frequencies between 125 and $500 \mathrm{~Hz}$ (A, white noise; $\mathrm{B}$, weighted noise; $\mathrm{C}, 2 \mathrm{kHz}$ low-pass weighted noise). ( $\mathbf{\square}$, case 1; $\sqsubset$, case 2; $\mathrm{O}$, case 3-right; $\bullet$, case 3-left; $\triangle$, case 4-right; $\Delta$, case 4-left)

terms of the activation of the auditory nerve. Moreover, if there is a "saturation" phenomenon in the input mechanism of the AR, i.e., more input energy above some point is not effective to facilitate the $A R$, further input energy above that saturation point may not reduce the ART. In the present study, to test the former possibility, low frequency weighted noise was used and the results were compared between white and weighted noise. As shown in Figs. 2 and 3, no effects from changing the energy in the low frequency region were observed. On the other hand, to investigate the latter possiblity, the effects of a decrease of the activated frequency region were investigated by using a low-pass filter $(2000 \mathrm{~Hz}$ in the present study). If this "saturation" factor affects the phenomenon of a lesser contribution of the lower frequency energy on the ART of the filtered BBN, the effects of broadening of the passed frequency on the ART should be observed even below $700 \mathrm{~Hz}$ when using low-pass filtered noise at $2000 \mathrm{~Hz}$. However, no effects of broadening of the passed frequency below $700 \mathrm{~Hz}$ were observed in low-pass filtered noise, either.

The results obtained seem to indicate a lesser contribution from the frequency region below $700 \mathrm{~Hz}$ in the $\mathrm{AR}$ of human. This possibility is also suggested by 
the observation of the summed effects of two tones on the ARTs (Kawase et al. 1997). If this hypothesis is true, MEMs can contract most sensitively in response to broadband mid- to high-frequency stimuli, and the AR in response to low frequency sound below $500-700 \mathrm{~Hz}$ might be mainly elicited by upper frequency neurons exited by the mechanism of the spread of excitation (excitation of the tail part of the tuning curve of the high frequency neuron). These hypotheses are interesting in relation to the possible anti-masking role of this system; MEMs decrease the masking of responses to high-frequency sound produced by lowfrequency sounds as shown by the asymmetric character of the effects of the contraction of MEMs (Borg and Zakrisson 1974; Pang 1988), namely, the predominant reduction of sound transmission for the low-frequency region (Møller 1965; Pang and Peake 1986). That is, it seems that AR, basically, is elicited by the excitation of neurons innervated from the effective (anti-masked) region, in terms of this anti-masking role.

\section{Acknowledgment}

This study was supported by a grant from the Ministry of Education, Science, Sports and Culture (Grant-in-Aid for Scientific Research [C] 09671731).

\section{References}

1) Borg, E. (1972) Acoustic middle ear reflexes: A sensory-control system. Acta Otolaryngol. Suppl., 304, 1-34.

2) Borg, E. \& Zakrisson, J.-E. (1974) Stapedius reflex and monaural masking. Acta Otolaryngol., 78, 155-161.

3) Borg, E., Counter, S.A. \& Rosler, G. (1984) Theories of middle ear muscle function. In: The Acoustic Reflex, edited by S. Silman, Acad. Press., pp. 63-99.

4) Carmel, P.W. \& Starr, A. (1963) Acoustic and nonacoustic factors modifying middleear muscle activity in waking cats. J. Neurophysiol., 26, 598-616.

5) Dadson, R.S. \& King, J.H. (1952) A determination of the normal threshold of hearing and its relation to the standardization of audiometers. J. Laryngol. Otol., 66, $366-378$.

6) Deutsch, L.J. (1972) The threshold of the stapedius reflex for pure tone and noise stimuli. Acta Otolaryngol., 74, 248-251.

7) Djupesland, G. \& Zwislocki, J.J. (1973) On the critical band in the acoustic stapedius reflex. J. Acoust. Soc. Am., 54, 1157-1159.

8) Flottorp, G., Djupesland, G. \& Winther, F. (1971) The acoustic stapedius reflex in relation to critical bandwidth. J. Acoust. Soc. Am., 49, 457-461.

9) Kawase, T., Hidaka, H. \& Takasaka, T. (1997) Frequency summation observed in the human acoustic reflex. Hear. Res., 108, 37-45.

10) Klockhoff, I. (1961) Middle ear muscle reflexes in man. Acta Otolaryngol. Suppl., 164, 1-92.

11) Kobler, J.B., Guinan, J.J.Jr., Vacher, S.R. \& Norris, B.E. (1992) Acoustic reflex frequency selectivity in single stapedius motoneurons of the cat. J. Neurophysiol., 68, 807-817.

12) Margolis, R.H. \& Popelka, G.R. (1975) Loudness and the acoustic reflex. J. Acoust. Soc. Am., 58, 1330-1332. 
13) Margolis, R.H., Dubno, J.R. \& Wilson, R.H. (1980) Acoustic-reflex thresholds for noise stimuli. J. Acoust. Soc. Am., 68, 892-895.

14) Møller, A.R. (1965) An experimental study of the acoustic impedance of the middle ear and its transmission properties. Acta Otolaryngol., 60, 129-149.

15) Pang, X.D. \& Peake, W.T. (1986) How do contractions of the stapedius muscle alter the acoustic properties of the ear? In: Peripheral Auditory Mechanisms, edited by J.B. Allen, J.L. Hall, A. Hubbard, Springer Berlin, pp. 36-43.

16) Pang, X.D. (1988) Effects of stapedius-muscle contractions on masking of tone responses in the auditory nerve ( $\mathrm{PhD}$ dissertation). Cambridge, MA, MIT.

17) Peterson, J.L. \& Liden, G. (1972) Some static characteristics of the stapedial muscle reflex. Audiology, 11, 97-114.

18) Popelka, G.R., Margolis, R.H. \& Wiley, T.L. (1976) Effect of activating signal bandwidth on acoustic-reflex thresholds. J. Acoust. Soc. Am., 59, 153-159.

19) Robinson, D.W. \& Dadson, R.S. (1956) A redetermination of the equal-loudness relation for pure tones. British Journal of Applied Physics, 7, 166-181. 